\title{
Interference Resolving Radio Resource Allocation Scheme in a TDD-OFDMA/FDD-CDMA Hierarchical Overlay Cellular System
}

\author{
Yeonwoo Lee ${ }^{+}$, Kyung-Ho Kim ${ }^{++}$
}

\begin{abstract}
In order to support a cell-independent traffic asymmetry, the conventional TDD system cannot avoid crossed time slot (CTS) interference. Moreover, the TDD/FDD hierarchical overlay cellular systems is taken into account as a generally accepted cell model in a heterogeneous radio environment. In this paper, we propose an interference resolving radio resource allocation technique in a TDD-OFDMA cellular system that overlays a FDD-CDMA cell. In our proposed scheme, we exploit under-used FDD-CDMA uplink resource by TDD mobile abiding by a region based time slot(TS) allocation which in turn mitigates CTS interference considerably. It is demonstrated that combined with under-used resource utilization scheme based on mobile's location, the proposed technique can reduce CTS interference considerably and support the asymmetric traffic in TDD system.
\end{abstract}

Key words: Hierarchical overlay cellular, interference, TDD-OFDMA, FDD-CDMA, radio resource allocation

\section{INTRODUCTION}

In the latest mobile communication systems, the demand for multimedia services to support a high data rate and asymmetric traffic service has accelerated the need for interference robust transmission technique and resource management algorithm. Thus, an efficient and flexible resource allocation technique is key enabling technologies as

※ Corresponding Author: Yeonwoo Lee, Address : (534729) Mokpo National Univ. Dept. of Inform. \& Comm. Eng., 61, Dorim-Ri, Chunggye-Myon, Mjuan-Gun, Chonnam, TEL : +82-61-450-2745, FAX : +82-61-4506470, E-mail : ylee@mokpo.ac.kr

Receipt date: Apr. 1, 2013, Revision date: May 14, 2013 Approval date: June 4, 3012

${ }^{+}$Dept. of Inform. \& Comm. Eng., Mokpo National Univ. (E-mail: ylee@mokpo.ac.kr)

${ }^{+\dagger}$ Dept. of Inform. \& Comm. Eng., Mokpo National Univ. (E-mail:khkim@mokpo.ac.kr)

* This research was supported by Basic Science Research Program through the National Research Foundation of Korea(NRF) funded by the Ministry of Education, Science and Technology (Grant No.: KRF-2008-313D00648). regards enabling the flexible management of the various traffic requirements and maximal utilization of the radio resources in a system. Such technologies are very relevant to duplexing methods, i.e. time division duplex (TDD) and frequency division duplex (FDD) in $3 \mathrm{G}$ or $4 \mathrm{G}$ systems.

In TDD-based systems, the uplink (UL) and downlink (DL) signals are transmitted over the same frequency band, yet separate (multiple) time slots are assigned for the UL and DL transmissions, respectively. By adjusting the number of time slots assigned to each link, the TDD scheme can support asymmetric traffic demand between the UL and DL. Another major feature of the TDD system is channel reciprocity, which allows the system to adopt adaptive modulation and coding (AMC) and multi-input multi-output (MIMO) technology without an increase in the feedback information. In general, the TDD system can provide a higher trunk efficiency since the system utilizes entire bandwidth. In the TDD scheme, how ${ }^{-}$ ever, a guard time is required between the UL and 
DL intervals to prevent interference caused by the different round-trip delays among users distributed throughout the cell. In fact, more guard time is required as the cell size increases, reducing bandwidth efficiency. Therefore, the TDD system is more suited to data services in the short-range communication systems (micro or pico cell). Meanwhile, with the frequency division duplex (FDD) scheme, the UL and DL signals are transmitted using the different frequency bands separated by a guard band. However, since the frequency bandwidth is fixed, the FDD system is basically unable to support the traffic asymmetry between UL and DL. Nonetheless, the FDD scheme has no round-trip delay problem, in contrast to the TDD scheme. For these reasons, it would seem evident that the FDD should cover the macro-cell range, while the TDD should cover the $\mathrm{pico}^{-} / \mathrm{mi}^{-}$ cro-cell range, which was discussed as a topical issue in the $3 \mathrm{G}$, LTE-advanced, $4 \mathrm{G}$ hierarchical or overlay cellular networks [1-4].

A widely-used hierarchical TDD/FDD cell structure consists of an FDD underlay macro-cell and a TDD overlay micro- ${ }^{-}$cell. In such a system, most of previous works focused on adjacent channel interference (ACI) effect and its trade- ${ }^{-}$off de $^{-}$ pending on the optimal location deployment of TDD BS and FDD BS of the universal mobile telecommunications system (UMTS) [5]. Another interesting proposed work is a TDD/FDD underlay system proposed by [6] which exploits underused FDD-CDMA resource for TDD-CDMA pico-cellular users, increasing the flexibility of CDMA based TDD/FDD hierarchical cell structure. How ever, these previous works are constrained to CDMA based system with careful positioning of TDD-BS and FDD-BS.

Combined with the TDD providing a cell independent traffic asymmetry of uplink and downlink, OFDMA system that can support high-speed data rate service gains momentum for a future multimedia mobile communication system. How ${ }^{-}$ ever, when the TDD scheme is used for a multi-cell environment, it suffers from severe $\mathrm{CO}^{-}$ channel interferences (CCIs) between mobile stations (MSs) and base stations (BSs) when the frames are not synchronized with one another and/or each cell has a different UL/DL transmission timing. Such $\mathrm{co}^{-}$-channel interference appears in crossed time slots (CTS) where some cells are active in the downlink and other cells are in the uplink [5], especially at the cell boundary. Clearly, this CTS interference turns out major performance degradation factor has to be solved for achieving the full benefit of the TDD based system, i.e. traffic asymmetry.

Therefore, in order to fully exploit TDD sys ${ }^{-}$ tem's feature and efficient spectrum utilization functionality in a TDD-OFDMA cellular system overlaid a FDD-CDMA cellular system, we propose an efficient resource utilization technique us ${ }^{-}$ ing a region based TS allocation algorithm. Thus, we partition a cell into two regions (i.e. inner- and outer-cell region) where TDD-OFDMA system is utilized overlaying FDD-CDMA system. In particular, the outer-region users located at cell boundaries use both FDD-CDMA uplink and TDDOFDMA downlink. Moreover, we exploit underused FDD-CDMA uplink resource by TDD mobile abiding by a region based TS allocation which in turn mitigates CTS interference considerably. As such, the CTS interference is dramatically reduced and the outage performance significantly is improved with a simple TS allocation algorithm, which in result, providing asymmetric traffic service. This paper is organizes as follows. Section II describes the proposed interference resolving $\mathrm{ra}^{-}$ dio resource allocation scheme in a TDD-OFDMA/ FDD-CDMA hierarchical overlay cellular system. System level simulation with interference scenarios and discussion are presented in Section III, following by conclusion. 


\section{Proposed Interference Resolving Radio Resource Allocation Scheme}

\subsection{Interference Scenario in a Time Division Duplex(TDD) based Cellular System}

While the merits of a TDD based cellular system, i.e. an asymmetric traffic service supported by adopting different UL/DL time slot ratios between neighbor cells, should be fully utilized, this inherently entails TDD interferences. Fig. 1 depicts how these interferences occur in the TDD system. When cell 1 is in the downlink transmission period while the adjacent cell 2 is in the uplink period, each cell must transmit and receive the signal through an asynchronous time slot, i.e. crossed time slot (CTS). In such a case, a major interference can occur in the TDD system, i.e. a CTS interference, such as an MS-to-MS interference, BS-to-BS interference (i.e., same entity interference), or MS-to-BS interference (i.e., other entity interference), that degrade the system performance. As one of the solutions to mitigate its effect, the dynamic allocation of time-slots can be employed in a centralized or a distributed manner.

\subsection{Proposed Interference Resolving Time Slot Allocation Scheme}

The aim of the proposed radio resource allocation scheme for a TDD-OFDMA overlay cellular system is to provide asymmetric traffic services with resolving TDD inherent interference problem, i.e. CTS interference as shown in Figure 1, and thus, it provides more radio resources to mobile users. In order to resolve this CTS interference and utilize radio resource as well, we proposed the radio resource allocation scheme which is based on a mobile user's location in a TDD-OFDMA cell and a pre-defined frequency-time planning of a overall hierarchical cellular model. The proposed scheme is to prevent major interference-makers located at the cell boundary from using the same frequency band (TDD). That is, the proposed system $\mathrm{se}^{-}$ lectively allocates under-used FDD-CDMA uplink resource (codes) to users located at the cell boundary based on FDD-CDMA uplink codes availability, simultaneously accomplishing cell independent asymmetry in the TDD system. Note that it is assumed that a TDD-OFDMA mobile station set has dual-mode operation in a TDD/FDD overlay cellular system.

The idea of exploiting under-used FDD resource by TDD users is the same as in [5]. However, the main difference of the proposed system is to allow asynchronous TS overlap and to utilize cell independent traffic asymmetry, achieving the full flexibility of TDD feature. Note that ref. [5] does not allow asynchronous TS overlap and may not provide the full traffic asymmetry capability.

In the proposed overlay cellular system as shown in Fig. 2, cell coverage in the TDD-OFDMA system is partitioned into two (inner-and outerregion). For users at outer-region near cell boundaries, FDD-CDMA system and TDD-OFDMA
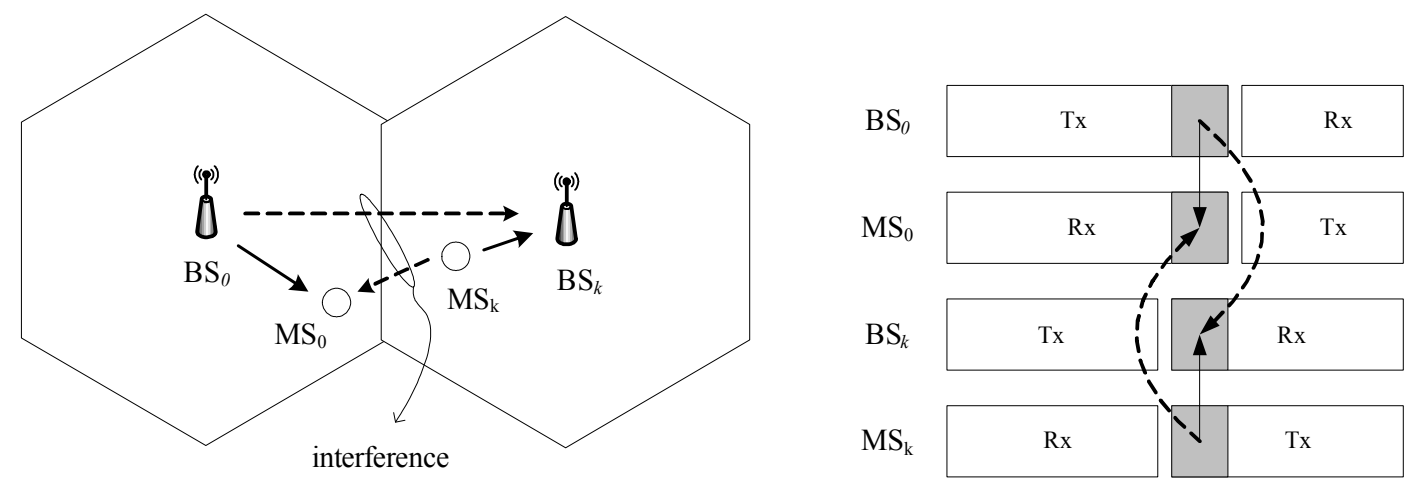

Fig. 1. Cross time slot (CTS) interference in a TDD based cellular system. 

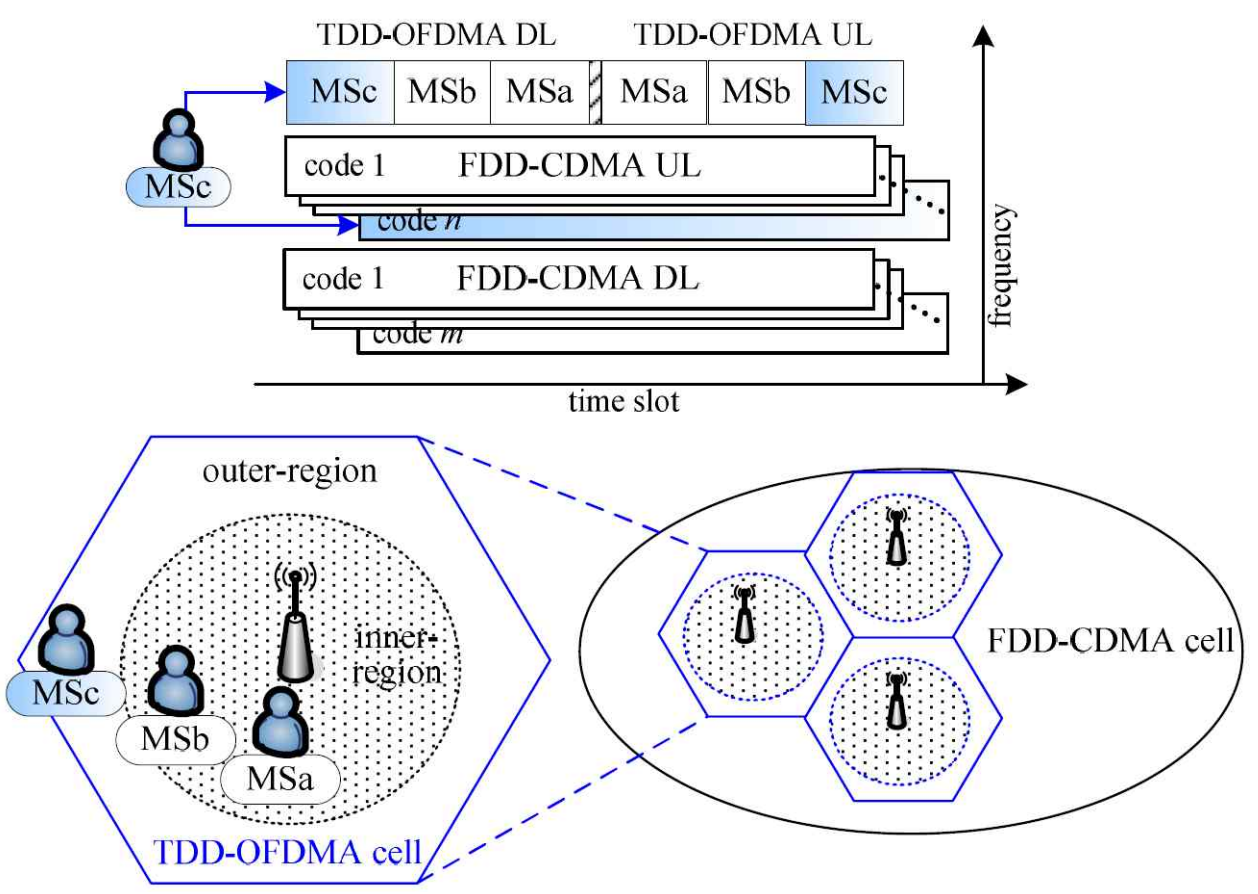

Fig. 2. Illustration of the proposed interference resolving TS allocation mechanism over a proposed TDD-OFDMA/FDD-CDMA hierarchical overlay cellular system.

system is used for uplink and downlink, respectively. Combined with the TS allocation algorithm based on region, this proposed system can achieve better performance improvement. This mitigates the severe cell-boundary inter-cell interference by combining the time slot allocation mechanism with the frequency allocation and the cell structure.

Although the proposed interference resolving TS allocation scheme over TDD/FDD overlay cellular system can also experience the same types of interference as in the TDD system, the amount of interference is different. In the proposed cellular system, the MS-to-MS interferences can be considerably reduced by employing FDD-UL codes at TDD outer zone, which enables most of the CTS interference victims to be isolated from interference coming from the MSs in the adjacent cells.

As shown in Fig. 2, for downlink of MS_a, MS_b at inner-region and MS_c at outer-region (in the order of closest to BS), TDD time slots closest to TS switching point are allocated to mobile users located at closer to BS, i.e. MS_a and MS_b. With this approach, the probability of CTS interference can be reduced since MS_c (which causes most of CTS interference) are not allocated into crossed time slots. For the uplink the algorithm follows the same principle, but it is applied only to MS_a and MS_b since MS_c located at outer-region are allocated to use FDD-CDMA uplink codes. Therefore, the proposed TS allocation algorithm can reduce inter-cell interferences and achieve the TDD flexibility. As shown in Fig. 2, TDD-OFDMA MS_c user can exploit FDD-CDMA uplink codes if these code-resources are available for borrowing. This simple procedure can resolve CTS interference caused by these TDD-OFDMA MS_c user.

More detailed structure of the proposed inference resolving TS allocation mechanism is shown in Fig. 3, where TDD-OFDMA MS_c user (i.e. TDD mobile user locating at TDD cell boundary) decided by a region based TS allocation is allowed to borrow a resource from FDD-CDMA UL resource (i.e. codes) if these codes are not occupied by other FDD users. 


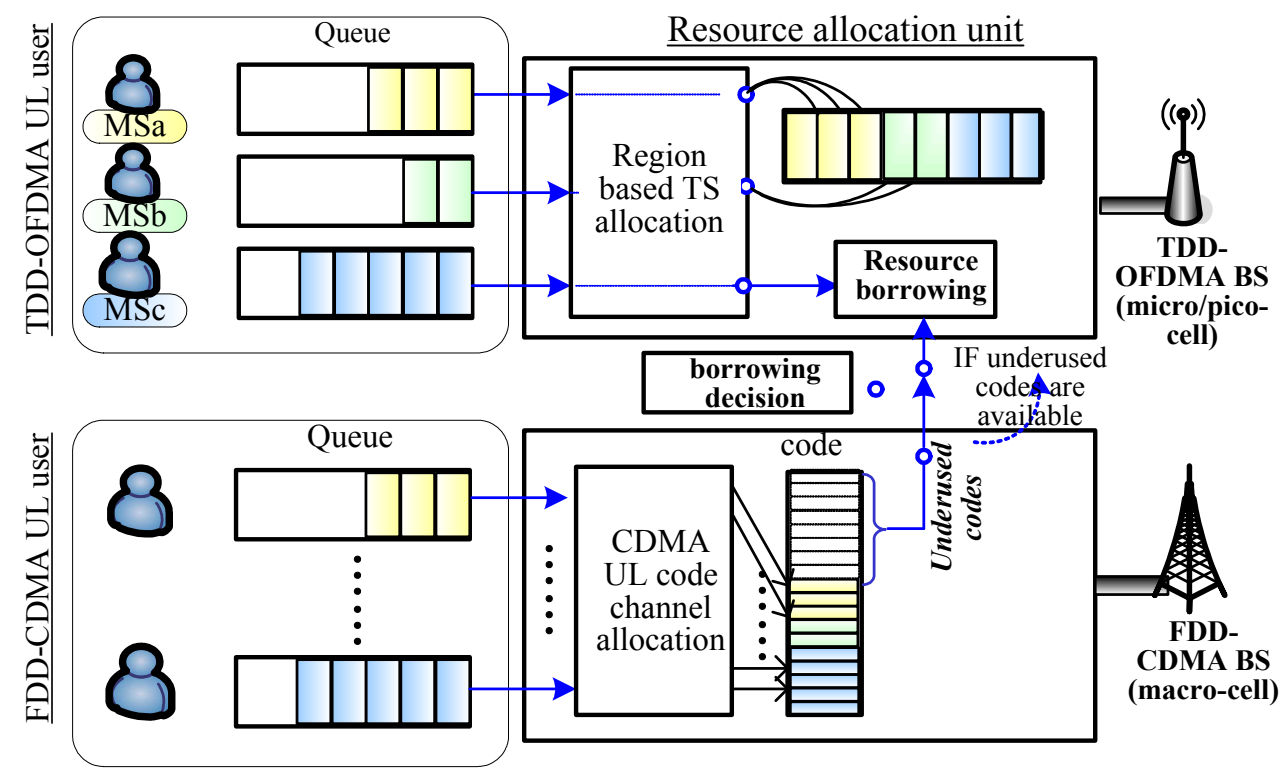

Fig. 3. Structure of the proposed interference resolving TS allocation mechanism projected to overlay cell system considering borrowing underused codes.

3. Simulation Results of Interference and Outage Probability Performance

3.1 CTS Interference Model and Interference Resolving Mechanism

In Fig. $4(\mathrm{~b}), M S_{0}^{\text {in }}(i), M S_{0}^{\text {out }}(i) M S_{0}^{\text {out }}\left(i^{\prime}\right), M S_{k}^{\text {in }}(j)$ and $M S_{k}^{\text {out }}\left(j^{\prime}\right)$ denote the $i^{\text {th }}$ MS in the inner zone and the outer zone of the reference cell and the $j^{\text {th }}$ MS in the inner zone and the outer zone of $\mathrm{k}^{\text {th }}$ cell, respectively. In the DL from $B S_{0}$ to $M S_{0} \mathrm{~s}$ during the CTS period, $M S_{0}$ suffer from interference caused by both $M S_{k}^{\text {in }}(j)$ and $M S_{k}^{\text {out }}\left(j^{\prime}\right)$ in the conventional TDD scheme. In the proposed TDDOFDMA/FDD-CDMA overlay cellular scheme, however, $M S_{0}^{\text {in }}(i)$ only suffers interference caused by $M S_{k}^{\text {in }}(j)$. For an UL case from $M S_{k}$ to $B S_{k}$ during the CTS period, $M S_{0}$ causes interference to $B S_{k}$ in both TDD scheme and HDD scheme. This implies that the proposed overlay cellular system can avoid the worst CTS interference while creating the TDD interference-free area by employing an FDD-UL region in the outer-cell area. Therefore,
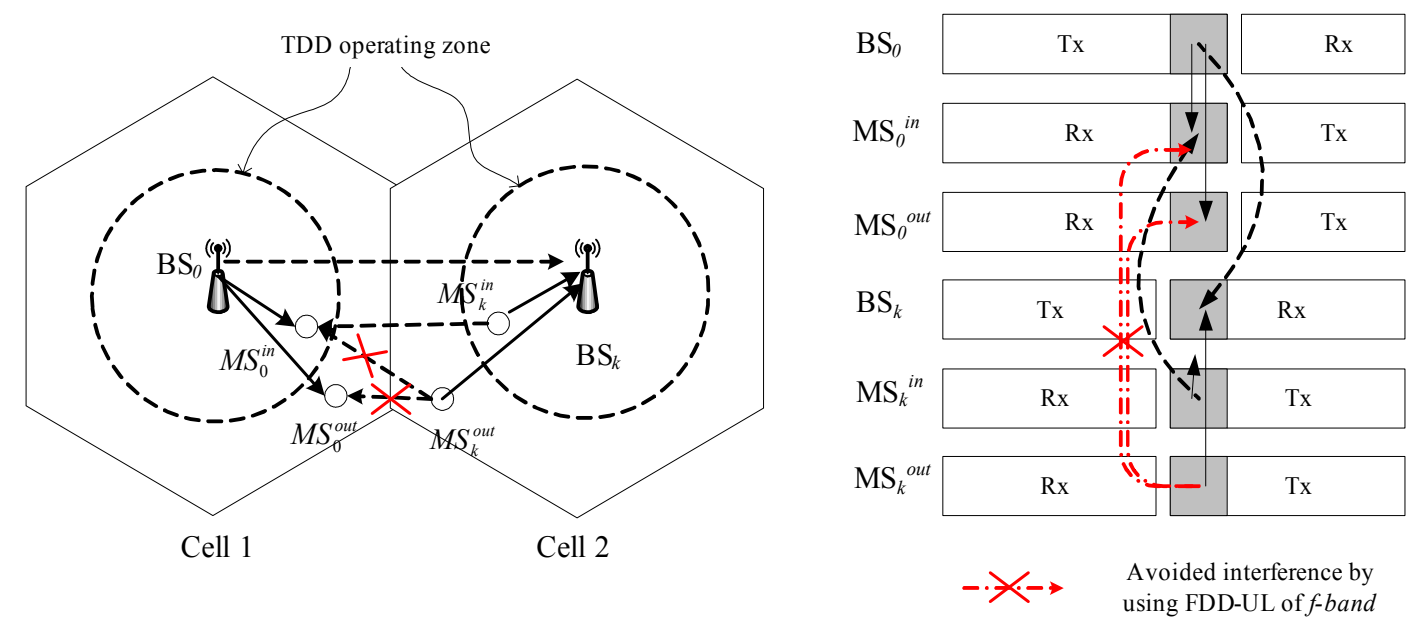

Fig. 4. Illustration of CTS interference in the considered overlay cellular system model. 
this structural concept significantly reduces the CCI between MSs. As a consequence, it enables each BS in the TDD system to operate with a variable asymmetric traffic ratio without suffering outage performance degradation at the outer-cell area.

\subsection{Simulation Parameters and Results}

In order to show the efficiency of the proposed method in terms of interference and outage probability performance of TDD-OFDMA overlay system, we evaluated the cumulative distributed function (CDF) versus signal-to-interference plus noise ratio (SINR) of the proposed system. We as ${ }^{-}$ sume frequency-hopping $(\mathrm{FH})$ TDD-OFDMA sys-

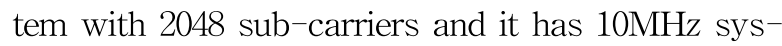
tem bandwidth at $2.3 \mathrm{GHz}$. The FDD-CDMA is assumed as having $1.25 \mathrm{MHz}$ bandwidth at $2.0 \mathrm{GHz}$ with processing gain of 64 and covers macro-cell. The cell-radius of TDD-OFDMA system is $1 \mathrm{Km}$ with inner-region of $700 \mathrm{~m}$. The simulated TDD cells are wrap-around $3-$ sectored 27 cells (3-tier) and 3 -sectored FDD cells are assumed to be un- derlay on TDD cells. The transmitting power from the $\mathrm{BS}$ and $\mathrm{MS}$ is set to $33 \mathrm{dBm}$ with $15 \mathrm{dBi}$ three-sector antenna gain and $23 \mathrm{dBm}$ with omni-directional antenna, respectively. The path-loss is considered using Log-distance model with the path-loss exponent 3.74 and the $\log$-normal fading with $10 \mathrm{~dB}$ standard deviation. For a frequency selective fading, ITU Veh-A channel model is considered [6],[7]. The generated number of users per cell is set to 48. System configuration and simulation parameters are shown in Table 1.

For CTS scenario, we randomly generate DL cells or UL cells according to the CTS cell ratio, i.e. $\{($ no. of $\mathrm{DL}$ cell $) /($ no. of $\mathrm{DL}$ cell + no. of $\mathrm{UL}$ cell)\}, and allocate CTS into inner-region user. We consider three CTS cell ratios such as 1/3, 1/2 and 2/3. Fig. 2 plots the downlink CDF versus SINR for the proposed system and conventional TDD system. As shown in Fig. 5, the proposed TDDOFDMA overlay system outperforms the conventional TDD-OFDMA system for various CTS cell ratios, $1 / 3,1 / 2$ and $2 / 3$.

We can observe that severe CTS interference is

Table 1. System configurations and simulation parameters

\begin{tabular}{|c|c|c|}
\hline Parameter & \multicolumn{2}{|l|}{ TDD } \\
\hline Carrier Frequency & \multicolumn{2}{|l|}{$5 \mathrm{GHz}$} \\
\hline System Bandwidth & \multicolumn{2}{|l|}{$10 \mathrm{MHz}$} \\
\hline Modulation Scheme & \multicolumn{2}{|l|}{ AMC } \\
\hline Multiple access & \multicolumn{2}{|l|}{ OFDMA } \\
\hline Frame Length & \multicolumn{2}{|l|}{$5 \mathrm{~ms}$} \\
\hline Number of slots per frame & \multicolumn{2}{|l|}{20 slots } \\
\hline \multirow{9}{*}{ OFDM } & FFT Size & 2048 \\
\hline & Sampling Frequency & $28.57 \mathrm{MHz}$ \\
\hline & Subcarrier Spacing & $13.95 \mathrm{kHz}$ \\
\hline & OFDM symbol length & 71.6835 us \\
\hline & Guard interval & 4.4802 us \\
\hline & Number of subchannels & $97(=1552 / 16)$ \\
\hline & $\begin{array}{l}\text { Allocation } \begin{array}{c}\text { Granularity } \\
\text { (symbol*subchannel/slot) }\end{array} \\
\end{array}$ & $\begin{array}{l}\text { DL: }(3 * 48) \\
\text { UL: }(3 * 16)\end{array}$ \\
\hline & Transmit power at BS & $5 \mathrm{~W} / 25 \mathrm{MHz}$ \\
\hline & Max. Transmit Power at MS & $250 \mathrm{~mW} / 25 \mathrm{MHz}$ \\
\hline \multirow{5}{*}{ CDMA } & Chip Rate & 3.84Mcps \\
\hline & Spreading Factor & 32 (Fixed) \\
\hline & Maximum No. of Codes/slot & 32 \\
\hline & Transmit power at BS & $1 \mathrm{~W} / 5 \mathrm{MHz}$ \\
\hline & Maximum Transmit power at MS & $200 \mathrm{~mW} / 5 \mathrm{MHz}$ \\
\hline
\end{tabular}




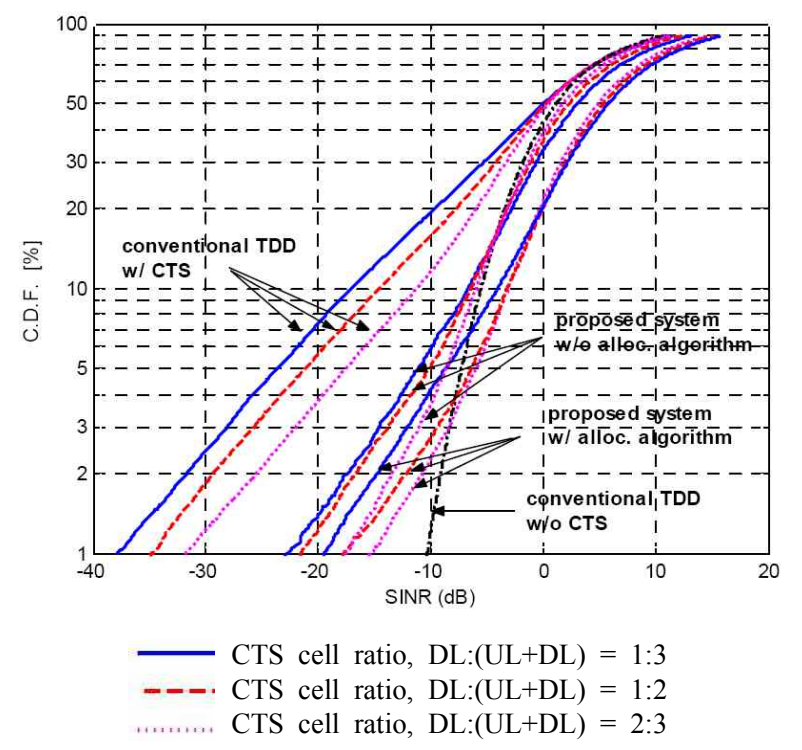

Fig. 5. Downlink CDF performance versus SINR as a function of CTS cell ratio.

considerably resolved. When the outage SINR level is set to about $-5 \mathrm{~dB}$, the proposed system without TS allocation algorithm can guarantee similar performance with conventional TDD system without CTS, but with allocation algorithm, it shows even further improvement. The uplink CDF performance is shown in Fig. 6 where the proposed overlay system shows dramatic performance improvement. Whereas the conventional TDD-OFDMA system

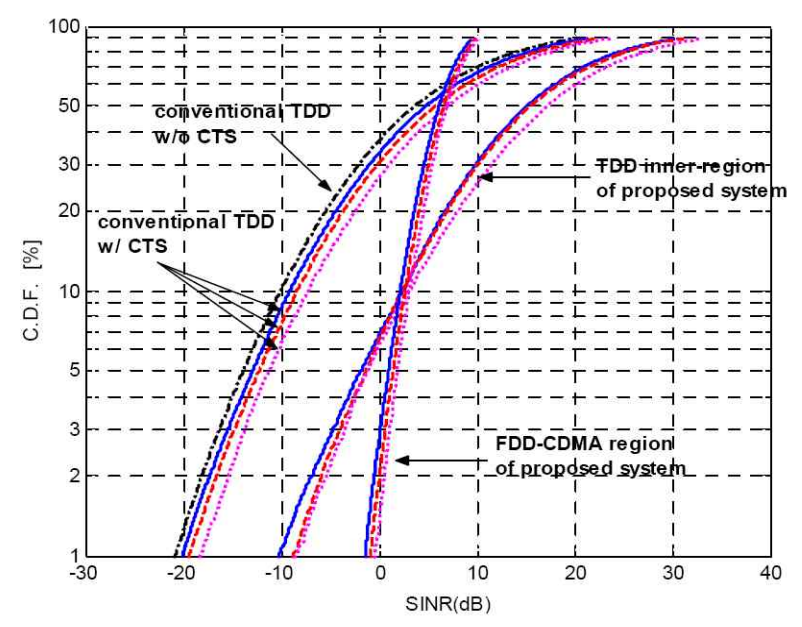

CTS cell ratio, DL:(UL+DL) $=1: 3$
-- CTS cell ratio, DL:(UL+DL) $=1: 2$
CTS cell ratio, DL:(UL+DL) $=2 \cdot 3$

Fig. 6. Uplink CDF performance versus SINR as a function of CTS cell ratio. suffers severe CTS interference, the TDDOFDMA uplink inner-region (MS_a and MS_b) and FDD-CDMA uplink outer-region (MS_c in Fig. 1) are free from CTS interference. Note that in the uplink system, the allocation algorithm effect is minimal because MS_c is assigned to FDDCDMA resource plane.

Moreover, we observe that in the conventional TDD uplink with CTS can show better performance than that without CTS, because the dominant interference in the uplink is caused by uplink mobile users (MS) rather than by downlink (BS). From both results, the proposed TDD-OFDMA overlay system with allocation algorithm can provide CTS (inter-cell) interference resolved TDD system.

\section{CONCLUSION}

In this paper, we propose an interference resolving radio resource allocation technique in a TDDOFDMA/FDD-CMDA hierarchical overlay cellular system. The proposed scheme exploits under-used FDD-CDMA uplink resource as an interference resolving mechanism by allocating TS based on TDD mobile users' region. It is demonstrated that when the overlay concept combined with CTS in ${ }^{-}$ terference resolving technique is applied to TDDOFDMA cellular systems, then a considerable CTS interference suppression and flexible traffic asymmetry can be obtained. With a simple TS allocation algorithm, we can expect further outage performance improvement.

\section{REFERENCES}

[1] G. Jeong and W.S. Jeon, "CDMA/TDD System for Wireless Multimedia Services with Traffic Unbalance Between Uplink and Downlink," IEEE Journal on Selected Areas in Communications, Vol. 17, No. 5, pp. 939946, 1999.

[2] Lili. Tang, "Performance Analysis of Mobile 
Communication in Picocell and Femtocell," Lecture Notes in Electrical Engineering, Vol. 225, No. 1, pp. 595-602, 2013.

[3] Sagari Shweta, Gautam Bhanage, and Dipankar Raychaudhuri, "Adaptive Geolocation based Interference Control for Hierarchical Cellular Network with Femtocells," Proc. Personal Indoor and Mobile Radio Communications, pp. 132-136, 2011.

[4] Hoang-Hiep Nguyen, Ngoc-Thai Pham, and Won-Joo Hwang, "Energy Efficient Base Station Operation in Heterogeneous Cellular Networks," Proc. the Korea Multimedia Society, pp. 158-159, 2012.

[5] H. Haas and G. Povey, "Capacity Analysis of A TDD Underlay Applicable to UMTS," Proc. Personal Indoor and Mobile Radio Communications, pp. 167-171, 1998.

[6] Recommendation ITU-R M.1225, Guidelines for Evaluation of Radio Transmission Technologies for IMT-2000, 1997.

[7] Ju-Phil and Choi, "Performance Evaluation of AMC in Clustered OFDM System," Journal of Korea Multimedia Society, Vol. 8, No. 12, pp. 1623-1630, 2005.

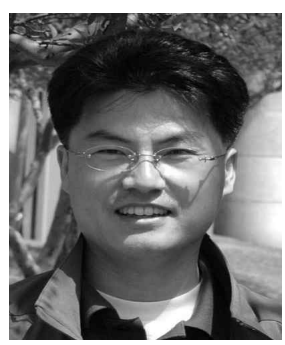

\section{Yeonwoo Lee}

is currently a Professor with the Dept. of Information and $\mathrm{Com}^{-}$ munications Eng. at the Mokpo National University, Mokpo, Chonnam, Korea, since Sep. 2005. He has been a senior researcher with $4 \mathrm{G}$ mobile com- $^{-}$ munication team at the Samsung Advanced Institute of Technology(SAIT) from Jan. 2004 to Aug. 2005. From Oct. 2000 to Dec. 2003, he has been a Research fellow with the School of Electronics and Engineering at the University of Edinburgh, UK. He received a MS and $\mathrm{Ph}$. D. in Dept. of Electronics Eng. from Korea University, Seoul, Korea, in 1994 and 2000, respectively. His research interests are wireless multimedia communications, cognitive radio, IT convergence technologies.

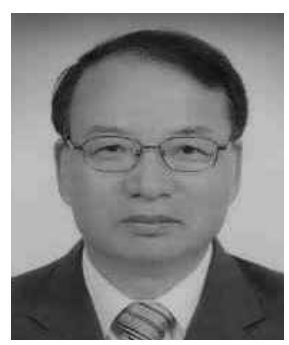

\section{Kyung-Ho Kim}

is currently a Professor with the Dept. of Information and $\mathrm{Com}^{-}$ munications Eng. at the Mokpo National University, Mokpo, Chonnam, Korea, since Mar. 1987. He received MS and Ph.D. in Dept. of Electronics Eng. from Chosun University in 1983 and 1993, respectively. He also has a Ph.D. from Howard University, USA in May 2000. His research interests are communication networks, data networks, system integration, next generation wireless communication network. 